\title{
The Archæology of Radical Pictoriality
}

Whitney Davis, University of California at Berkeley

Images are an empirical subject matter of art history. Indeed, in one sense they are the very subjects of art history: they are the noetic standpoints (usually correlated with real spatial and temporal locations) at which art-historical objects such as pictures and artworks are realized by human agents, that is, made visible to them in visual space. (To be sure, the mere visibility of a picture or artwork is not sufficient to constitute it as recognized or recognizable in a 'visuality', that is, as having recognizable 'forms of likeness', whether directly visible and visual or not, within which the representation has specifically cultural intelligibility.) But such images are not the artifactual objects of art history. The artifactual objects in question are pictures or artworks that must be imaged by people. ${ }^{1}$

In this essay I consider the relay - the feedback - between human subjects who image (and can make pictures) and material configurations that picture (and can be imaged). Despite common parlance in art history, I distinguish, then, between images and pictures. We see pictures; we look at them, looking for something in them. But we do not see images. In images we see, say, a painting and perhaps the picture it relays, or the picture as painted. For we see with images. Better, we image tout court: we see the visible world as we naturally see it, including any pictures that might be visually afforded to us in it. As a function of the physics and psychophysics of the visual field, human imaging conforms to the laws of light and geometrical optics as the primate nervous system has adapted to them in hominid evolution. But it does not only conform to the laws of light, luminance, and the visual angle in the ecologies to which it is habituated. It also works recursions in them, 
unbinding them from the primary or first-order reflexes of photoreception and as it were 'autonomizing' them: namely, the recursions of what I will call 'radical pictoriality', which need not always produce material artifacts such as drawings or paintings, and in turn possibly of depiction. Of course, historians of visual culture, especially traditions of specifically pictorial art, chiefly address themselves to depiction. But depiction itself, I will argue, cannot be fully understood without considering the primary and often prior recursions of radical pictoriality specifically in imaging.

\section{1 \\ Radical Pictoriality}

In successions of radical pictoriality, or the autonomic capacity of natural vision to virtualize what it sees, we revisibilize aspects of things in the very activity of seeing them, visually remaking them into something else (or sometimes bigger or brighter or closer), whether or not these activities succeed to depiction (Table 1).

Table 1. Basic Model of Radical Pictoriality in the Succession of Imaging

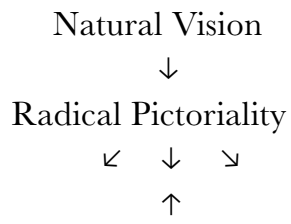

Pictorialization

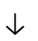

Depiction

Consider the famous Müller-Lyer illusion, in which two parallel lines that are equal in length appear to be unequal (Fig. 1): I see the equal lines as unequal. In the usual grapheme that relays the illusion, in fact, the top line $\mathrm{AB}$ appears to be quite a bit longer than the bottom line $\mathrm{CD}$ (a feature of the 
Fig. 1

A grapheme of the Müller-Lyer Illusion.

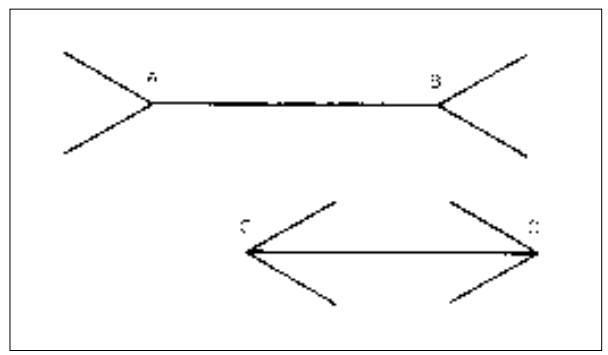

illusion not always spelled out explicitly), though exactly how much longer or longer in just what way might be difficult to say even if a difference in length seems plain to see.

I can try to see the illusion itself - the fact that the two unequal lines are really equal. That is, I can try to see what I see these features of the grapheme as - equal lines as unequal - as equal. I might do this geometrically by setting the figure into a grid that shows me that the lines have the same lengths (Fig. 2), that is, by making a kind of picture of the optical stimulus, the grapheme itself. I might be able simply to visualize this configuration. But likely I will have to draw it in order to see it well enough to confirm that it shows the salient equality of the two lengths. (The result could be recomputed perspectivally to turn a visual and graphic demonstration in plane or ordinary geometry into one in natural geometrical optics [Fig. 3], though once the visible metric equality of the grid-squares is lost in the perspective configuration, if it is, the geometric proof of the 'illusion' will also become invisible, as does the illusion when 'in perspective'.)
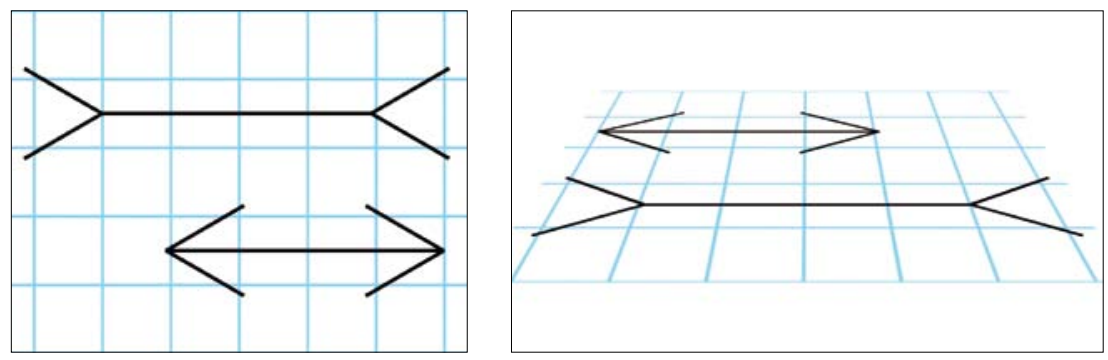

Figs. 2 \& 3 A grapheme of the Müller-Lyer Illusion (2) set in a grid, (3) in 'perspective' rendering. 
Without drawing anything at all, however, I can also image the lines pictorially, giving pictoriality to them by virtualizing - by revisibilizing — what I see the lines as (namely, unequal in length) as the lengths of something-or-other in particular: $\mathrm{AB}$ as, say, the bottom keel of a short canoe seen from just below and CD as, say, the top of a huge skyscraper seen from high above (call this Fig. 1': it cannot, of course, be readily illustrated in any printing of the grapheme, though it could possibly be pictured by me in a drawing of it). Now it might seem to me that it is $C D$ that is the longer line and $A B$ that is the shorter. I have visibilized a certain particular unequalness in the grapheme seen as having unequal lines: its unequalness is the unequalness of certain particular lengths I have virtualized, namely, the short canoe and the huge skyscraper. This 'radical pictoriality', as I will call it, is quite distinct imagistically from my imaging of the grapheme in the first-order reflexes of vision, for there the lines, though unequal, were quite differently so visually (i.e., AB > CD). The new virtuality is 'radical' because it is bound neither to the real configuration of the lines, which are actually equal, nor to anything that they really depict, for the grapheme does not depict anything at all and I am completely free to see any number of other things in the lines. ${ }^{2}$

Of course, I have not disambiguated the fact that $\mathrm{AB}$ and $\mathrm{CD}$ are equal from the sensation that they are unequal. But I have reduced or reversed the illusion, or more exactly what I first saw the grapheme as. (Ex hypotheosi I did not initially see the illusion per se-see the grapheme as the illusion it is.) For in the Müller-Lyer illusion as I reflexively see it, it is CD that seems shorter than $\mathrm{AB}$ - not $\mathrm{AB}$ shorter than $\mathrm{CD}$, as in my radical-pictorial reimaging. Or at least I have reduced or reversed an illusion, namely, the grapheme seen as just the relation between the lines that is staged visually in the Müller-Lyer illusion specifically, an event of 'seeing-as' that has the deleterious property of being quite wrong metrically.

It would seem, then, that I can image two readily differentiated kinds of illusion (radical-pictorial imagistic virtualizations) in the grapheme: one array in which $\mathrm{AB}$ is visibly longer (the Müller-Lyer illusion as it is usually described) and one array in which it is visibly shorter (my radical pictorialization) (Table 2).

Put another way, though I can see the stimulus in only one reflexive way, that is, the way in which the illusion overrides the metric 'reality', nevertheless the grapheme has at least two radical-pictorial horizons. More exactly, it has two indefinitely large sets of the radical pictoriality of the unequal lengths of the 
Table 2. Radical Pictoriality in the Müller-Lyer Illusion and Its Variants

Grapheme

$\downarrow$

Natural Vision $\mid \leftarrow$ Radical Pictoriality

$<\quad \downarrow \quad \vee$

(A) $\{$ M-L illusions $[\mathrm{AB}>\mathrm{CD}]\} \mid(\mathrm{B})\{$ non-M-L illusions $[\mathrm{AB}<\mathrm{CD}\}\} \mid(\mathrm{C})\{$ no illusion $[\mathrm{AB}=\mathrm{CD}]\}$

$\downarrow \quad \downarrow \quad \downarrow \quad \downarrow \quad \downarrow \quad \downarrow \quad \downarrow \quad \downarrow \quad \downarrow$

Fig. 1 ? ? Fig. $1^{\prime}$ ? ? $\quad$ Fig. 2 Fig. ?

two lines (assume, again, that I do not see them to be equal), namely, (A) $\{\mathrm{AB}$ $>\mathrm{CD}\}$ (the illusion usually described) and $(\mathrm{B})\{\mathrm{AB}<\mathrm{CD}\}$ (the illusion as I can revisibilize it in radical-pictorial imaging). Moreover, if I can visibilize a proof of their equality (e. g., Fig. 2), arguably I can go on to populate set $\mathrm{C}\{\mathrm{AB}=$ $\mathrm{CD}\}$ with radical pictoriality recursively derived from measuring or diagramming the stimulus.

Of course, our underlying visual ability to discriminate any one line as visibly shorter or longer than any other must have neurophysiological limits, usually called visual 'acuity'. Still, my acuity does not limit my active visual dealings with the configuration as it is visible to me, that is, with whatever degree of acuity. Certainly it is sufficiently sharp and resolved for me to virtualize many different lengths in lines of just the visible size and width of Fig. 1. But we must remain open to the possibility, natural or cultural or both, that some people could see more finely unequal line-lengths than I can (or less), and therefore (1) that they could visibilize aspects of the lines, or kinds of radical pictoriality in the lines, that must be invisible to me and (2) that they could coordinate and communicate them in depiction. ${ }^{3}$ By the same token, their practices in depiction recursively might enable them to see more (or less) finely unequal line-lengths than I can. 


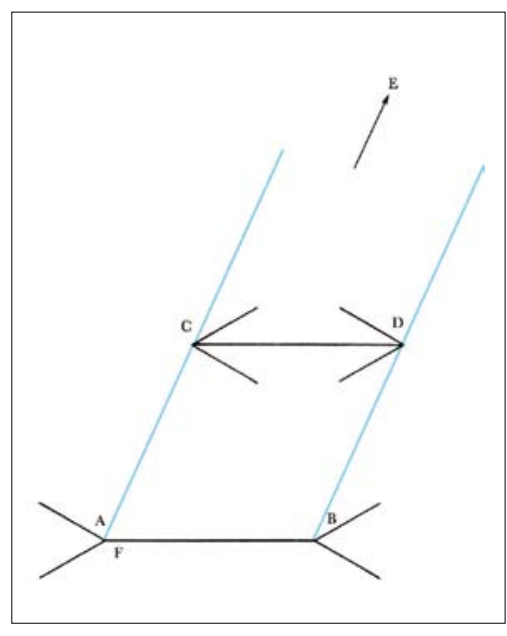

Fig. 4

A grapheme of the

Müller-Lyer Illusion pictorialized as transversals in a 'perspective' projection (vanishing point at E).

For the moment, what counts analytically is that the two sets of differently visible unequal lines, (A) and (B), remain empty until I actively populate them with radical-pictorial members: revisibilizations of particular inequalities in the lengths of the lines to which I succeed visually by virtualizing them as the lengths of something-or-other I can see them as, a visual 'succession' from the first-order reflex in which I already see the grapheme as some one member in (A) $\{\mathrm{AB}>\mathrm{CD}\}$. Regardless of neurophysiological and psychophysical limits on visual acuity in imaging the lines as visibly longer or shorter, there would seem to be no salient limits on radical pictoriality in imaging a line, whatever its visible length, as having the length of something-or-other in particular. For the fact that in $(\mathrm{B})\{\mathrm{AB}<\mathrm{CD}\}$ I can image $\mathrm{AB}$ as the keel of a canoe and image $\mathrm{CD}$ as the top of a skyscraper, revisibilizing $(\mathrm{A})\{\mathrm{AB}>\mathrm{CD}\}$ in a newly particular virtualization, does not prevent me in $(\mathrm{B})$ from reimaging $\mathrm{AB}$ and $\mathrm{CD}$ as things other than canoe and skyscraper while still virtualizing $C D$ as longer than $A B$ in just that particular relative length (that is, the relative length virtualized in [B] $\{\mathrm{AB}$-canoe $<\mathrm{CD}$-skyscraper $\}$ ), and regardless of my ability to measure the proportion (as in [G]).

Radical pictoriality is not limited by acuity at all (though what can be seen at all is a condition of radical-pictorial revisibilization). It is limited only by visual 'imagination' - the autonomization of autonomic or reflexive imaging, as I would like to put it, in active self-virtualizing recursions of human vision. 
Fig. 5

A geometric proof of the nonconvergence of 'receding' lines (i.e., 'orthogonals' converging at $\mathrm{E}$ ) in Figure 4.

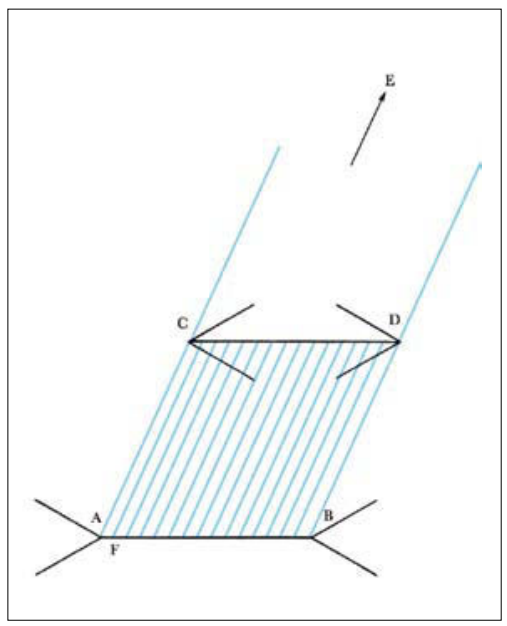

Thus there are as many members of $(B)\{A B>C D)$ for me as there can be radical-pictorial images of the relation between $\mathrm{AB}$ and $\mathrm{CD}$ virtualized by me and revisible to me in the configuration relative to my first-order reflex in spontaneously seeing the configuration as some one member in the set (A) $\{\mathrm{AB}>\mathrm{CD}\}$. In sum, though the illusion of inequality is an insuperable fact of the reflexive visibility of the configuration to me, it is a malleable matrix for my imagination of it. This is especially true if radical pictoriality eventually succeeds to depiction as such.

For example, recalling Fig. 3, picture $\mathrm{AB}$ and $\mathrm{CD}$ as same-size transversal ties on receding railroad tracks converging perspectivally at $\mathrm{E}$, a far point on the right side of a virtual horizon line in the 'distance' (Fig. 4). The tracks could not really converge. (I might picture this fact of optics and plane geometry as such [Fig. 5], as I did in picturing the fact of the equality of the length of the lines [Fig. 2].) But in imaging it can seem that they do precisely because the ties seem unequal. Under the perspective pictorialization, if I can succeed in visibilizing the grapheme in this way, I see the unequal lines as equal-equal in transversal length in their unequalness in the spatial recession that I have virtualized.

Again, picture the lines $\mathrm{CA}$ and $\mathrm{DB}$ as two of the four edges (with $\mathrm{AB}$ and $\mathrm{CD}$ ) of a rectangular tabletop (albeit a very rickety one!) that we want to set up (Fig. 6, overleaf). While quadrilateral, this tabletop cannot really be 


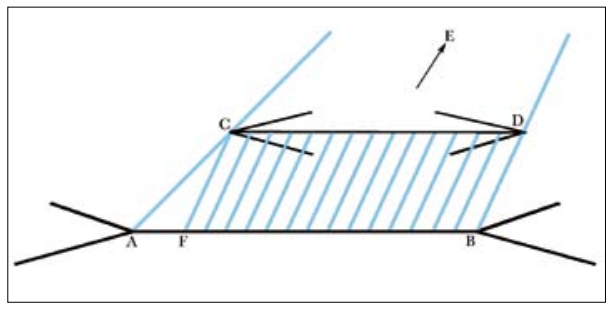

Fig. 6

$A B, C D, C A$, and $D B$ in a grapheme of the Müller-Lyer Illusion pictorialized as edges of a 'rectangular' tabletop ABCD. (Note CAF as a geometric proof of its nonrectangularity.)

rectangular, yet another fact that I could picture (e.g., in the 'proof' visible in $\mathrm{CAF}$ ). But when I admit the radical pictoriality of this tabletopness in the imaging of $A B C D$, it can seem that way. For in this pictorialization $A B$ and $\mathrm{CD}$ can be seen as exactly-the-same-in-length as sides-of-the-rectangle in which CA and DB are visibly the same length.

In turn, I might depict this tricky tabletopness (or at any rate a painter like Paul Cézanne might be able to do so), preserving and even heightening our visual sense of having interacting or compound views of the table in rectangular plan, in quasi-isometric diagrams of its sidedness, and in a perspectival recession. Here I might graphically set out certain pictorialities both of the illusion of linear inequality (partly as it can be 'perspectived') (Fig. 3) and of the plane (Euclidean) geometry of rectangular areas (including the way they can be perspectived) (Figs. 5, 6), so long as I image the picture itself under a visual angle that deviates no more than about forty degrees from an axis perpendicular to the surface. ${ }^{4}$ For radical pictoriality, of course, is a visual parameter of depiction in both the imaging that succeeds to depiction (Table 1) and the imaging that investigates it (see Table 5).

\section{2}

\section{Seeing-As, Seeing-In, and 'Seeing-As- $A s$ '}

As my use of the language of Wittgenstein's aspect psychology might have suggested, radical pictoriality is not so much a visual affordance of things (the apparent unequalness of $\mathrm{AB}$ and $\mathrm{CD}$ in the interaction between the stimulus and our perceptual habits) as it is a visible aspect of things (the particular longness or shortness of $\mathrm{AB}$ relative to $\mathrm{CD}$ ). It certainly enrolls an awareness that we are seeing something as something - a line as shorter because it has canoe-likeness 
(or whatever) for us. But this should be distinguished, as Table 3 suggests, from first-order 'seeing-as' understood to be the default reflexive condition of vision: simply seeing the rabbit or the duck, for example, when presented visually with the 'duck/rabbit grapheme' popularized by Joseph Jastrow (Fig. 7, overleaf) and cited in Wittgenstein's Philosophical Investigations; seeing short AB or long $\mathrm{AB}$; and so on. ${ }^{5}$

Table 3. Basic Model of Recursions of 'Seeing-As' in Radical Pictoriality

\section{Configuration}

(Duck/Rabbit Grapheme; Müller-Lyer Grapheme; etc.)

$\uparrow$

\section{Natural Vision ('Seeing-As')}

Duck/Rabbit as duck or rabbit; Müller-Lyer as AB > CD or AB < CD; etc.

$\downarrow$

\section{Radical Pictoriality ('Seeing-As- $A s$ ')}

$k \quad \downarrow \quad \succ$

$\operatorname{duck}(\mathrm{AB}>\mathrm{CD}$; etc. $)$

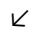

Seeing-as-as-as ...

K

white duck in the shadow

$\uparrow$ rabbit (AB < CD; etc.)

$\searrow$

Seeing-as-as-as ...

$y$

black rabbit splashed by light $\uparrow$

Pictorialization ('Seeing-In')

$\downarrow$

Depiction

For everything must be seen as something in particular; all affordances are apprehended as things with certain aspects, often readily recognizable, most usually as a visible world of recognizable objects. (Certainly they are not seen as what they must be optically at retinal photoreception: a luminance gradient, a distribution of differentially lighter and brighter 'pixels'. Or if they are 


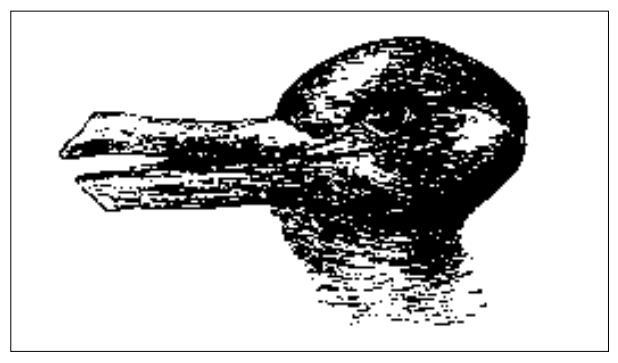

Fig. 7

The Duck/Rabbit grapheme.

From Joseph Jastrow,

Fact and Fable in Psychology

(London: Macmillan, 1901), 295.

so seen sometimes, it is in a radical pictoriality of the visible - not in firstorder seeing.) Having seen rabbit, on another occasion, of course, I might see duck. But the point of identifying radical pictoriality as a succession and recursion in first-order seeing-as (Table 3 ) is that on the very occasion of seeing the rabbit (or duck) I can visually work to see a duck (or rabbit) - an effort in which I might succeed or might fail, presuming that I undertake it in the very first place.

Indeed, the oscillation of the two seemingly stable pictures embedded in the duck-rabbit grapheme (often described as a reflexive 'Gestalt switch' between rabbit and duck) is really incidental to this point. It is just one of many possible active outcomes of radical pictoriality in imaging the configuration. In fact, it is an outcome that has been carefully staged graphically in the grapheme, which 'tricks' the eye in the manner of a true optical illusion (though it is not). Its configured dual depictiveness (i. e., depicted duck and depicted rabbit have been pictorially managed to be coextensive and thus entirely 'switchable' or fully substitutable) recursively affects one horizon of radical pictoriality (i. e., duck can be revisibilized as rabbit, or vice versa, partly in virtue of the dual depictiveness of the grapheme as configured-of its specifically pictorial 'bivirtuality').

But we should not mistake this effect of depiction with radical pictoriality tout court. In inspecting the grapheme, and regardless of what I immediately see it as and what I might know it to be qua depiction (however unitary, dual, or multiple), I could just as well work visually to see a white duck as a black one (what I happen to see the duck I see in the grapheme to be) or a duck with a rabbity eye or a rabbit with a feathery neck, whether or not whiteness, a rabbity eye, or a feathery neck are depicted, that is, whether or not the duck I see the configuration as being is depicted to be white. (Indeed, I radically 
pictorialize these things precisely because the configuration can be taken - can be seen as - depictively ambiguous about whether the duck is white or black, or both, or neither.) Moreover, and equally important, I could work to see neither any duck (white or black) nor any rabbit in the duck-rabbit grapheme. Instead I could work to see a head of garlic or a sprouting onion, to name radical-pictorial virtualities that the grapheme can have for me despite my fair certainty that it does not depict these things at all.

In the usual account of the phenomenology of the duck/rabbit grapheme, of course, I do not see the rabbit in the duck, but the duck/rabbit grapheme as rabbit or duck. That is, I see rabbit (one depicted object) or duck (another depicted object) in the duck/rabbit configuration. (As noted, however, I might see whiteness in the duck [it is seen-in as a white duck, despite the many black areas of the configuration], or, indeed, the rabbitiness in its eye.) In a widely-read essay, Richard Wollheim called this involution by the name 'seeingin'. In virtue of what he took to be the 'twofoldness' of pictorial representation (and perhaps overly impressed by the dually-depictive configuration of the duck/rabbit grapheme), seeing-in was specifically localized by Wollheim in our imaging of depictions. ('Twofoldness' refers to the joint if sometimes oscillating visibility of graphic configuration on the one hand [in this case, the duck/ rabbit grapheme as a dually-depictive configuration] and the virtual objects depicted on the other hand [in this case, duck or rabbit]). To be sure, the duck/rabbit grapheme is not, perhaps, a very good example of twofoldness in Wollheim's sense. Precisely because of its dual depictiveness, we might tend to see it only as duck or rabbit; that is, duck or rabbit is seen in the configuration ('seen in'), which has little or no visibility other than in the two pictures it stage-manages graphically (when it is not duck it is rabbit, and vice versa). Or if it does, these visibilities have rarely been remarked in commentary, though the pictorialist had to consider them. ${ }^{6}$

If I see a rabbit in the grapheme, I can also work visually to see it as, for example, a white rabbit in the shade or a black rabbit with a white spot on its nose (short $\mathrm{AB}$ or long $\mathrm{AB}$; etc.) (Table 3): the configuration can be differently (re)visibilized depending on the radical pictoriality the object seenin the configuration (what I see the duck-rabbit grapheme as) has for me. In the white rabbit, the spot on the nose is a patch of fur, but in the black rabbit, it is a splash of light - radical pictoriality that I can transfer to the $\operatorname{duck}(\mathrm{s})$ I can revisibilize as well. (Is the white spot a splash of light falling on dark feathers beside the duck's eye, or feathers of the light-colored duck visible 
just beyond the shadow falling on it? I can work to see the grapheme seen-as a duck in either way, working to see the dark- or light-colored ducks in it.) With Wollheim, I can properly say that I see a (depicted) light-splashed black rabbit in the grapheme when I see it as a rabbit. But the point here is that I could work to see it as a (depicted) white-spotted rabbit too. If successful, I would now see white-spottedness in the black rabbit where I had seen it as light-splashed. Seeing-in constitutes a particular 'pictorialization' in the configuration - the white-spotting in the configuration that I see as a rabbit (or duck) pictorializes the color of fur (or feathers) or the brightness of light (in relation to patches of shade). But this pictorialization is secured in the face of the radical pictoriality of the configuration when seen-as that pictorialization. For I could see the pictorialization as other things too.

As previous paragraphs might have suggested, then, radical pictoriality in my sense could be defined as 'seeing-as-as'. However something is seen-as (for everything, as already noted, must be seen as something), I can see it as something with new aspects - aspects perhaps sufficient to constitute a different thing for me to recognize. (Of course, I need not do so, for in seeing-as I already see something in particular, albeit not in the particular way I might revisibilize it.) And I can see that something as something, and so on, in relays of seeing-asas-as, seeing-as-as-as-as, etc. These locutions, however clumsy, enable us to notice the specifically recursive involution of radical pictoriality in imaging whether or not we are imaging pictures and whether or not depiction recursively affects $i t$. When I see the line $\mathrm{AB}$ as shorter than $\mathrm{CD}$, I am seeing $\mathrm{AB}$ as, say, canoelike; if I did not see AB as canoe-like (seeing-as-as), then perhaps AB would be seen as longer than CD.

'Seeing-as-as' does not depend on 'twofoldness' in Wollheim's sense, even if 'seeing-in' may devolve from our conjoint ('twofold') visual awareness of the drawn or painted mark (such as a 'long' or 'short' line) and the object it depicts (such as the keel of a canoe). For seeing canoe-likeness in AB-seen-asshorter-than- $\mathrm{CD}$ does not mean $\mathrm{AB}$ depicts a canoe, though sometimes $\mathrm{AB}$ could do so. It means that $\mathrm{AB}$ can have certain respects of canoe-likeness for me, whether or not I render the keel of a certain canoe in the line $\mathrm{AB}$ when I draw it and, if I do render this canoe, whether or not I depict these very aspects of it. Radical pictoriality precedes depiction, even though there may be recursions in which depiction may contribute it to natural vision when, for example, I see a tabletop as as-if-painted-by-Cézanne, a radical pictoriality of that 'still life' for me. 
In sum, radical pictoriality reconstitutes objects that are already visible, revisibilizing them in a particular visual virtualization. As Wittgenstein might have said, it puts them into a new light by actively assigning new visual aspects to them, not only allowing but visually enabling them to 'dawn' on us. ${ }^{7}$ This work does not supersede seeing-as, or differ from it in kind. It is its continued active ramification, including its emergence into our awareness of it - a recursive autonomization (as I have put it) of imaging that might be called 'æsthetic' (and certainly is 'imaginative') though it is not restricted to the efforts we make in seeing artworks. Stated another way, though radical pictoriality is a reflexive adaptation of vision, it is not the first-order reflex of reflected light stimulating the receptors of the visual cortex and usually issuing in the visual computation of recognizable objects (seeing-as). Rather it is a reflex that can arise as a response to it, as it were rereflexive - the self-recursions of our recognition of objects. For in recognizing objects, we sometimes seek to re-cognize them by revisibilizing them.

\section{3 \\ Depiction and the Imaging of Pictures}

I can readily see a smudge in the sky as a cloud, or even as having certain aspects of a camel (I see the smudge as a cloud-camel). And in radical pictoriality, I can try to see the cloud as a camel (not just a cloud), or, as may be, the cloud-camel I see the smudge in the sky to be as dark (not light) or very large and far away (not smaller and closer) (as-as). As the visual activity of virtualizing seeing-as, radical pictoriality can be said to 'pictorialize' things: we visually make things have a virtual aspect for us in visual space. Of course, the smudge/cloud is constantly changing in visual space, whether because of its own inherent mutability or because of mine, or both together. Indeed, I could cease to see it as a cloud; perhaps the smudge in the sky is a smear on my glasses, though this is not what I had initially seen it as. And with or without having virtualized a cloud-camel (as-as), I could virtualize other things too-revisibilizing the smudge, whether cloud or smear, as something other than a camel (as-as-as). What, then, if I take a visual interest in one virtuality that I have visibilized, specifically looking out for $i t$ ?

What I have been calling 'pictorialization' (Table 1) is the succession of radical pictoriality in its revisibilization - its revirtualization. Having once seen 
a particular camel in a cloud (I have virtualized the cloud in that particular radical-pictorial way), I can try to see it in the clouds, which are never quite the same, wherever and whenever I glance up at the sky. I can try visually to make any cloud have cloud-camelness - the very cloud-camelness, in fact, that I have seen. And I can try to see the short-canoe-like $\mathrm{AB}$ (one radical-pictorial member of the set $[\mathrm{B}]\{\mathrm{AB}<\mathrm{CD}\})$ in the Müller-Lyer illusion every time I encounter it, despite differences in the way the grapheme might be drawn or printed; and try to see the white-spotted rabbit-in-the-shade in the duck/ rabbit grapheme (it might be easy to see in Jastrow's variant of the grapheme [Fig. 7] but less easy visually to revirtualize in less detailed versions); and so on. If radical pictoriality (seeing-as-as) is rereflexive with respect to the firstorder reflex of seeing-as, pictorialization might be described as transreflexive ('seeing-as-as-in') and as its increasing autonomization and hence its increasing intentionalization: it involves active selection of radical pictoriality, even a selection among radical pictorialities, as if the very field of visibility were 'twofold' at least. A cloud-camel with particular aspects becomes my autonomous visual object even as I am visually aware (aware precisely in virtue of the radical pictoriality that $I$ have constituted) that the cloud is not really a camel and that the camel could be other than it is. I have pictorialized a cloud-camel as something to be seen in the world even if the world contains no such thing visible outside the radical-pictorial recursion. ${ }^{8}$

In turn, such pictorialization becomes depictive - it precipitates a depiction (Table 1) — when I visibilize something specifically in order for a particular virtuality to be seen in it: when I image the cloud just in order to see the cloud-camel that I can make it become, despite its other visibilities and possible virtualities. To be sure, art historians and other students of visual culture do not ordinarily address purely visual depictions, that is, depictions constructed purely in visual imagination, whether it is engaged in 'seeing' or not. Instead they address depictions materially produced for visual imagination, notably the recursions described here. But such artifacts must be constituted in imaginative imaging. And that can be intrinsically depictive when it envisions things in the world in order to see what they virtualize. I need make no artifact for my seeing to depict. For I can certainly use the cloud-camel that I can visibilize in the cloud as a way specifically to see a camel-perhaps, in fact, the only way to see just it.

Depiction becomes a picture in the received art-historical sense, a physical artifact that displays a virtual world rendered for and displayed to beholders, 
when I replicate these successions not only visually but also materially. Probably I cannot actually make a cloud. But I can make a cloud-camel in any number of ways. Indeed, as soon as I make a single visible mark on a surface I have done everything that is materially necessary for the entire successionrecursion of depiction to occur. To give a complete model of the succession (Table 4), it is usually my manipulations of materials and media, especially of marks, that have stimulated the autonomization of imaging described in this essay. The entire succession is readily triggered by making marks that pictorialize in our imaging of them - that have radical pictoriality (whether one virtuality or more) that we can recursively see the marks as rendering, or can visibilize them to do. And all marks can do so to the extent that they are autonomous affordances in the visual environment. We can transmute their 'standing out' into 'standing for'.

Table 4. Complete Model of Successions of Pictoriality in Imaging

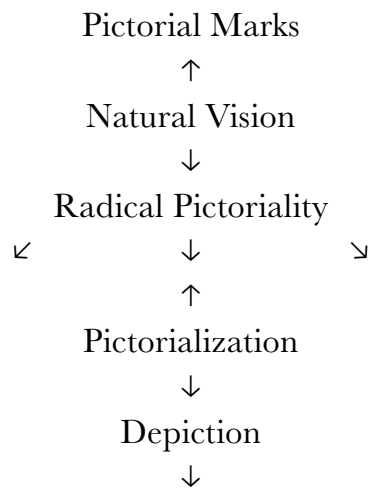

Mere Picture

Still, even an artifact made as pictorial need not actually depict, or be depictive. To depict is to coordinate a particular autonomized pictoriality in someone's imaging of things - one's own or others' - and for that imaging in the sense already considered, that is, for its own sake in virtualizing something. 
In making material marks or working material shapes, one can set out to do this. One does it by creating, resolving, selecting, sorting, and replicating radical pictoriality in a succession that leads to its visible virtualization: we see it as the virtuality that the configuration has been produced to support. And one can often be successful. But many pictures fail to pictorialize or render anything to beholders, especially those who do not succeed to the picture's visuality or visual culture.

What I call a 'mere picture', then, is a depictively-marked artifact awaiting someone's imaging of its pictoriality, including the investigation and resolution of radical pictoriality, not depictive, that the configuration-the affordance - might be imaged to have. This imaging might attain the depiction the artifact was intended to have. But it might not. To adapt a term from Simon Blackburn's philosophy of language, a mere picture can be 'wooden': morphologically it is a picture (and has no other causal explanation), but it has no pictoriality, at least for a particular beholder pictorializing it at a given time and place. An unknown quotient of depiction in human history (likely a vast quotient) probably is partly or even wholly wooden for some or many beholders, including the very makers of the pictures in question. Therefore it must be admitted that many pictures (when 'mere pictures' in the sense adumbrated here) do not fully belong to visual culture; they cannot be fully seen by people who image them to have the visible virtualities that have been deliberately made to be seen by way of them. ${ }^{9}$

Of course, the virtual visibles also need to be really visible. So far I have described the precipitation of depiction in the 'pictorializing' successions of the radical pictoriality of natural vision. But if a picture is indeed created, it becomes a material affordance to seeing - and it must be imaged. Obviously, then, and as I have already noted in passing several times, we must consider the imaging of pictures. As already suggested, the radical pictoriality of mere pictures must be pictorialized in a certain way in order to relay the coordinated depiction, for the picture was made as a coordination of a certain pictorialization that could be seen in it and that ideally it should be seen-as-as. Detailed exposition of this process is unnecessary. As Table 5 indicates, it can be modeled as symmetrically inverse to the process of pictorialization modeled in Tables 1 and 4 (though it is, of course, a recursion within it). Natural vision images mere pictures - even marks and shapes - in such a way as to pictorialize them despite nondepictive radical pictoriality that we can make in response to them. For we can see marks and mere pictures as many things 
(and see-in and see-as-as with respect to them) without at all seeing the things (or without seeing all the things) they depict.

Table 5. Complete Model of the Succession of Imaging Pictures

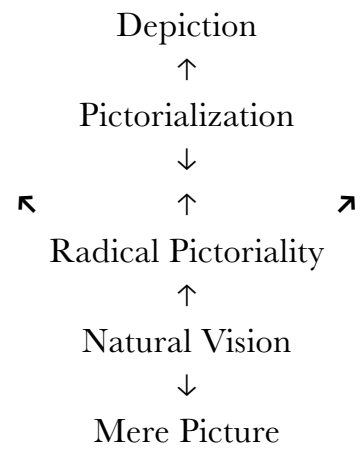

4

The Archæology of 'Image Games'

In the last two sections of this essay, I indicate two of the main 'archæological' implications of the conceptual considerations presented above.

For more than twenty millennia, modern humans have been training themselves to make discriminations in creating, preserving, and modifying visual virtualities - discriminations of affordance and aspect, of radical pictoriality and depiction, of one imaginable pictoriality or depiction in a mere picture and other pictorialities, and so on (and as set out in Tables 1-5). They often use material devices to help them do so. Some of these are pictures, though many pictures also build in numerical computers to guide imaging in appropriate directions or toward the right features (Table 5): notations of count, sequence, and grouping; scales and proportional ratios; effects of lighting, coloring, and foreshortening calculated to specify values of virtual luminance or spatiality. ${ }^{10}$ 
Requiring refined discriminations within the successions of pictoriality, pictures have come to have many social uses in legitimation, in ritual, and in relationality, and especially in communication. But it would not be surprising if one primary use of some pictures - perhaps the primary use - lies simply in flexing visual imagination, refining its reflexes and enhancing its depth, range, and power. A picture need be nothing but a pure 'image game' to do this. Indeed, imaging every picture (whatever the picture might do socially when imaged appropriately) requires that its image game be played. Even the most highly conventionalized pictures used in communication, such as pictograms (for example, in hieroglyphs) or icons (for example, in 'pictorial languages' such as Otto Neurath's Isotype or other visual codings), presume image games and gaming. For even the most highly conventionalized communicative icon can have unpredictable quotients of radical pictoriality in our visual space. As in speaking with another person in the frame of a 'language game', in an image game I see what is meant (if I do so at all) by noticing how what is visible to me is being made and used (or was made and used) to do and to show certain things.

Every visual culture likely must produce some pictures (or use parts of certain pictures or successions of pictoriality) to show the game itself (or the games), and allow users to play it (or them) more or less freely. (In fact, every single picture must have some way to indicate what game it plays.) To this end, certain pictures may teach the broadest range of image gaming - of successions of pictoriality, radical and otherwise - that can be imaginable in that culture at that time, defining the very possibility and limits of visual culture and visuality. They need not be rarified products of fine art, though some rarified arts may have acquired or appropriated the function of pure image gaming. Instead they are likely to be ubiquitous and easy to make (even if they are not visually simple) as well as pleasing and perhaps puzzling. At heart they allow beholders visually to learn about the successions of pictoriality that constitute any mere pictures they may see. They rehearse the successions of visibilization and virtualization that made them, allowing beholders continually to make and remake them in imaging. As we have seen, they will do this anyway. But in an image game they can do it recursively, noticing the particular forms of likeness accepted in the game.

The groups of engraved 'plaquettes' from the Magdalenian sites of La Marche (Vienne), Saut-du-Perron (Loire), Gönnersdorf (Rhineland), and elsewhere may be examples of pure image games in prehistoric material culture. ${ }^{11}$ 
Especially at Gönnersdorf, the plaquettes often display complex networks of lines crossing one another and, if closely inspected, therefore visibly lying 'over' or 'under' one another. Pictures emerge in these networks; they are also often superimposed, and often over- or under-engraved with many other lines. Though I cannot make the demonstration here, I have little doubt that the engravings were specifically made to be imaged as palimpsests of visible marks and emergent pictures, even though (indeed precisely because) not all of the visible marks seem to function pictorially (though their radical-pictorial potential is clear, and could easily be activated in handling) and no visible picture enrolls all the marks in its pictoriality (at least so far as we can identify the depictions).

The social functions of the plaquettes are unknown. They have sometimes been thought to have had a 'ritual' use-meaning; because many of them were deliberately broken, it has been suggested that the engravings, pictorial or not, were ritually 'killed'. ${ }^{12}$ Regardless, their rapid production (experiments suggest they could be made in a few minutes), great quantity (many dozens have been recovered from the sites mentioned), multiple makership (many different 'hands' have been identified in the engravings), repetitive iconography (though still formally and stylistically differentiated), and self-reiterating or re-marking graphism (sometimes called 'double-lining') suggest pure image-gaming.

There may well have been 'Gestalt switches' (as in the duck/rabbit grapheme) between some embedded pictures. But the plaquettes also seem to construct graphically interconnected pictorialities that cannot be switched. Some pictures on the plaquettes share some of the same lines, for example, but can only be imaged - pictorially visibilized - in inverse planes of the plaquette, that is, in being viewed from different orientations and angles. (Similar multiplications of standpoint occur in cave painting.) Though seemingly simple, and probably created in the visual coincidences and convergences of the autonomic successions of pictoriality, these constructions had to have been refined deliberately - a fact that the beholder must come to see as such. (Specifically, the beholder comes to see that the graphic morphologies, the configurative constructions, could have had no other cause; as noted, however, I will not offer forensic demonstration of this here.) In inspecting and handling them (and no doubt talking about them), one could - one now still can - recognize, reintegrate, and revisibilize some of the pictorialities that had emerged in producing them (though probably not all), comparing, compositing, recombining, sorting, and maybe counting the many fragments of lines, figures, planes, and 
surfaces - a testing of acuity, memory, and visual-pictorial flexibility, of imaging or imagination itself. Indeed, the image games of the plaquettes might have been games in the most literal sense.

\section{5 The Archæology of Standpoints}

As the Magdalenian plaquettes suggest, we disambiguate the radical pictoriality of pictures for us and attain the depiction (if we do) at a standpoint. It can be defined in part as a particular place or zone of imaging pictures in real and visual space.

Precisely because it is the arena of the constitution of a visual virtual world, pictorialists usually try to remove obstacles to this physical standpoint (or in it), regardless of its real (metric) distance from the mere picture. (Many pictures completely lack depiction when imaged from too far away or too close; though self-evident, the point is often overlooked, as if the image that constitutes the picture, or pictoriality, is always simply 'in' the artifact.) At the standpoint ideally we see the picture just as (and as-as ...) what was made just to be seen in it. To be sure, as I have insisted all along, this picture will have the radical pictoriality (if any) of the pictorialization we can see there, along with the radical pictoriality (if any) of the artifact that relays it, the mere picture. In natural vision it cannot not have these essential horizons of visual ambiguity and æsthetic richness. But ideally it has no others. Or if it has, they can be (re)visibilized to be consistent with the things seen to be virtualized in the picture specifically.

In a theoretical definition, then, pictorial standpoint is the notional identity of pictorialization in depiction with radical pictoriality in imaging it - the fully transitive mutual recursion of the inverse successions (Tables 4 and 5). It is the place of visuality, the standpoint at which we can visibilize things as they were visually constituted to be virtual for us - to have certain forms of likeness - or where we see them 'as they were meant to be seen', to use a more colloquial formula. In this sense, visuality cannot, of course, be a mere physical place only, though a place is required for what is virtual in visuality to be visible in vision. In real and visual space, visuality is a noetic standpoint as well: the competence of the image maker in the image games of the visual cultures he traverses socially. 
Still, as a matter of method - of the anthropology of imaging - we must often begin with the physical place of imaging the picture, especially its optics. For as beholders of the mere picture who can generate our own radical pictorializations of it as an object afforded in our visual field, we can easily be excluded from the visuality in which the depiction was coordinated - exclude ourselves in virtue of our radical-pictorial activity in imaging the picture. Ideally Tables 4 and 5 are fully transitive mutual recursions. But empirically they might not be. In fact, usually they are not, even if the slippage or disparity is not salient in many social uses of depiction, often coordinated in advance to take account of inevitable disjunctions in real standpoints of imaging them. (Certainly a pictorialist can anticipate that no two beholders will image the depiction at exactly the same physical location at exactly the same time.) This is not just a matter of so-called cultural difference - of not belonging to the visuality of the pictorialist. It is chiefly a matter of historical distance - of being unable fully to review and revisibilize the successions of radical pictoriality and pictorialization that were originally coordinated in a depiction even if we ourselves have produced it. What remains of this 'lost' historical visuality, its archæological fossil as it were, can only be radical-pictorial parameters of the mere picture that are partly preserved at standpoint in virtue of the inherent geometrical optics of natural vision at that place.

For historians of pictorialisms in Western art since the later Middle Ages, these considerations may seem trivial. The physical standpoints for the depictions in question - their orientations, our sightlines, the visual-angular conformation of pictorializations coordinated therein-are usually well known, organized in standardized formats, genres, and types. Moreover, even if visuality at said standpoints is not a dominant visual parameter of our form of life now, it remains part of our inheritance; in virtue of the successions modeled here, it is built in to recurrent and even current radical pictoriality, pictorialization, and depiction. Many of us still know how to organize standpoint in looking at altarpieces, gallery pictures, or public sculptures. If we do not, it is easy to find out how. The architectonics of standpoint may still be visible in situ. But depart from such accessible contexts of visuality and our visual confidence dissipates.

We do not know, for example, how the engravers of La Marche visually encountered the feline predators of the Vienne, let alone a particular such beast (perhaps in part imagined or fictive) that they seem to have pictorialized. ${ }^{13}$ Nor do we even know how they saw their pictures of it. The plaquettes were 


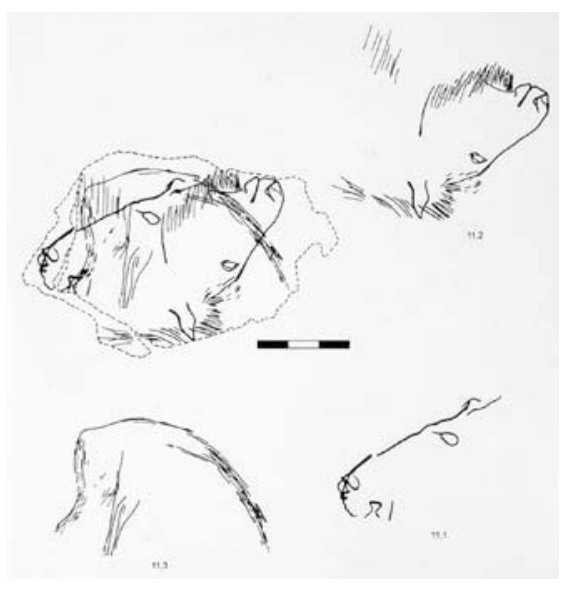

Fig. 8

Plaquette 11 from Magdalenian site at Andernach, Rhineland, Germany. From Gerhard Bosinski, Tierdarstellungen von Gönnersdorf: Nachträge zu Mammut und Pferde sowie die übrigen Tierdarstellungen (Mainz: Verlag des RömischGermanischen Zentralmuseums, 2008), Abb. 11. Reprint by permission.

found disordered as detritus (a secondary reuse in a revetment for a hearth), even if they had been made and used (possibly around the hearth itself) in the well-lighted rock shelter in which they were found. Mixed with chunks of quartz, quartzite, and hematite and with fragments of animal bone, the plaquettes of Gönnersdorf were found in thematic 'concentrations' or clusters on the floor of a circular structure, probably covered; it seems to have been seasonally occupied by two different human groups who are thought to have traveled to the site from different regions. ${ }^{14}$ One of the activities in the shelter may have involved handling the plaquettes, possibly in a social group gathered near the firepit and by passing the objects around. (Eventually, however, they seem to have been laid down as a kind of flooring.) Inherently this situation of standpoint enabled a turning or 'spinning' of the pictures that would involve angles of vision - that is, imaging them - from many sides. In fact, it would seem to have required it.

In fact, we do not always need archæological evidence for real physical standpoint, as at Gönnersdorf, to infer constitutive visuality at standpoint, and in turn hypothetically to identify the original physical place or zone of imaging. The visible reorientability or multiorientationality of the Magdalenian plaquettes persists for us as an optical parameter of the mere pictures - a material residue of their use at standpoint, where they were likely visibilized as pictorial palimpsests in the kind of image game described already, that is, configured in imaging successions in just this activity of rotation at standpoint, and only in this activity. 
For example, if we turn or 'spin' Plaquette 11 from Andernach (Rhineland) (Fig. 8) we find that a 'third' head can emerge, quite different from the horse's head visible in profile in one view and, in the plane 180-degrees inverse to it (or 'upside down'), another profile horse's head: a dog's- or wolf-like beast's head rendered from 'above', as if we are looking down upon it. (It can be seen, then, whether one looks at the plaquette 'upright' or 'upside down' with respect to the other figures.) Oddly, the excavators did not see (or at least did not record) this third head as one of the depictions visible on the plaquette; perhaps they reasoned that if it could be seen from all vantages it was not deliberately made to be seen at one, as with the other figures. For me, it virtualizes in striking volume, modeling (hatching), and even foreshortening - so striking that perhaps the excavators rejected it as a possible prehistoric pictorialization and took it simply to be radical pictoriality on their part, if they visibilized it at all. ${ }^{15}$ But notice the graphic calibrations that had to have been pursued in order to constitute it even as a merely radical-pictorial horizon of the marks coincident with it: For instance, the righthand leg of the mammoth's hindquarters that is visible in the 'upright' view creates the lefthand 'ear' of the emergent dog's- or wolf-like head (though it is in the plane inverse to it) even as it graphically replicates the horse's ear that is visible in the same orientation; by contrast, the hatching of the muzzle of the emergent dog's- or wolf-like head creates the ruff of the horse visible in the 'upside-down' view as well as the mane of the horse visible in 'upright' view. (Or vice versa in both cases.) Because its relations to other lines and figures can only be seen in each case from different vantages, the dog's- or wolf-like head must have been stage-managed; in this image game, it was put there to be seen as having been pictorialized as depiction in just this way, and specifically in visible tension with the possibility that it is merely a radical pictoriality on our part, if we see it at all. Thus we can succeed appropriately from its radical pictoriality to its depictiveness even as the former dynamizes the latter in the image game. No other causal account of the visual generation, organization, and configuration of the marks, qua marks, makes archæological sense of what is plainly visible.

If this standpoint can be architectonically and performatively localized in a set-up like the circular shelter erected around the fire pit at Gönnersdorf, so much the better. But it is not essential. It could be that the plaquette workedstage-managed its ambiguous palimpsest - whenever and wherever it could be reimaged in the way described, that is, wherever its game of rotation is played. The optics of radical pictoriality in natural vision are continuous enough between the past and the present for us to infer this standpointed 
visuality: given the graphic coordination of the emergent head, the plaquette from Andernach could not not have had visual activation in multiple reorientations and rotations.

In this sense an archæology of image games and an archæology of standpoints complement one another in the project of locating radical pictoriality within the relevant historical horizons - as a succession in the making of pictures in the past or as a succession in imaging them today, and, of course, in both. 


\section{Notes}

1 I discuss seeing the 'forms of likeness' of things, or 'visuality', in A General Theory of Visual Culture (Princeton: Princeton University Press, 2011). I argue there that visuality - seeing the forms of likeness of things, or the network of analogies within which they are culturally intelligible - is partly produced in interaction with pictures, even though all pictures need not be exclusively intelligible within visuality (they can merely be visible to vision).

2 I use the modifier 'radical' in the term 'radical pictoriality' with analogy to its sense in atomic chemistry, not in politics. Every electron in the outer shell in an atom (e. g., oxygen) in the ground state is paired with an electron spinning oppositely. In a free radical, however, the atom has at least one unpaired electron in the outer shell, and can have independent existence (e. g., hydrogen peroxide).

3 'There could be people who recognize a polygon with ninety-seven angles at first glance, and without counting', as Wittgenstein wrote, possibly referring to Archimedes's method for finding the value of $\pi$ (the ratio of the circumference to the diameter of a circle) by using a ninety-six-sided polygon inscribed in the circle (Remarks on the Philosophy of Psychology, vol. 2, edited by G. H. von Wright and Heikki Nyman, translated by G. G. Luckhardt and M. A. E. Aue [Oxford: Blackwell, 1980], 79e, no. 433). This possibility underwrites strong historicist claims in art history and visual-cultural studies. But it could also be fully compatible with 'naturalist' claims for variations in vision - for its speciation. For discussion, see Davis, A General Theory of Visual Culture, 11-41, 187-92.

4 Many basic software programs for digital-image processing allow simulations of this rotation. At too great an oblique angle of viewing the rectangular picture, CAF will not be seen as lying 'in' the table, as what has been pictured about it; it may be seen instead as a mere effect or by-product of the beholder's obliquity. It becomes a radical pictoriality of imaging the picture (see Table 5) as distinct from a radical pictoriality in the imaging recoordinated as the depiction to be seen. To be sure, this further recursion cannot simply be ignored. To control it, pictorialists usually require imaging to occur at a particular physical standpoint.

5 Though perhaps a merely editorial slip, Wittgenstein may have used a reduced version of the grapheme, eliminating the interior hatching, that had been published by Jastrow in Fact and Fable in Psychology (London: Macmillan, 1901), 295. Perhaps this is one reason why his discussion overlooked certain striking recursions of ambiguous pictoriality in the grapheme, or so it seems to me.

6 See Richard Wollheim, Art and Its Objects, second edition (Cambridge: Cambridge 
University Press, 1985), 205-26; see also Davis, A General Theory of Visual Culture, 344 n. 32. Wollheim's analysis has been refined by many other writers. Dominic McIver Lopes, for example, has proposed a typology of seeing-in that sorts its behavior with respect to different kinds of pictorial arrays (see Sight and Sensibility: Evaluating Pictures [Oxford: Clarendon, 2005]). These include troublesome trompes l'œil that visually do not have to have the 'twofold' pictorial structure that supports seeing-in (as distinct from seeing-as) in the seeing of pictures as the visible interaction of a marked surface and virtual (depicted) objects. Here I cannot discuss Wollheim's philosophy of pictorial representation and organization in relation to his psychoanalytic (Kleinian) philosophy of mind; see Whitney Davis, 'Freudianism, Formalism, and Richard Wollheim', in Queer Beauty: Sexuality and Aesthetics from Winckelmann to Freud and Beyond (New York: Columbia University Press, 2010), 271-95.

7 Ludwig Wittgenstein, On Certainty, edited by G. E. M. Anscombe and G. H. von Wright, translated by Denis Paul and G. E. M. Anscombe (Oxford: Blackwell, 1969), 21. For one possible use of Wittgenstein's metaphor in visual-cultural studies, see Davis, A General Theory of Visual Culture, 36-37, 326-28, 337-38. Of course, Wittgenstein did not discuss the 'dawning' of aspects as 'radical pictoriality' - the active visual revisibilization of aspects seen. Radical pictoriality in my sense is one route of the dawning of aspects. There may be others.

8 This account has affinities with Kendall L. Walton's theory of pictorial representation as a species of 'make-believe' (Mimesis as Make-Believe: On the Foundations of the Representational Arts [Cambridge, MA: Harvard University Press, 1980]) and with models in ethology, psychology, and elsewhere of the evolution of 'pretence'. My approach differs from Walton's insofar as I take radical pictoriality and pictorialization to be activities in natural vision, though autonomizing it - to be the 'makebelieve' of human seeing as such, of which the 'make-believe' of depiction is one recursion. But obviously the construction of depictions immeasurably contributes to the imaginativeness of vision.

9 See Davis, A General Theory of Visual Culture, 216-29 (I use the example of Édouard Manet's Olympia as exhibited in the Paris Salon of 1865), 324-26.

10 For examples, see Whitney Davis, Masking the Blow: The Scene of Representation in Late Prehistoric Egyptian Art (Berkeley and Los Angeles: University of California Press, 1992), which discusses 'ciphers' within pictures that enable them to be handled and imaged in the right way, and A General Theory of Visual Culture, 120-49, on notations in pictures that specify their imaging standpoint because they are only 'readable' there. Such 'ciphers' and 'compunotations' in depictions are not the 
same thing as scripted text conjoined with pictures, for these often do not indicate how to image what the picture depicts.

11 The principal recent sources (all superbly detailed) are Léon Pâles and Marie Tassin de Saint Péreuse, Les gravures de la Marche, Pt. 1, Félins et ours, 2 vols. (Bordeaux: Dalmas, 1969); Ann Sieveking, Engraved Magdalenian Plaquettes: A Regional and Stylistic Analysis of Stone, Bone, and Antler Plaquettes from Upper Paleolithic Sites in France and Cantabric Spain (Oxford: British Archaeological Reports, 1987), and Les plaquettes de schiste gravées du Saut-du-Perron (Oxford: British Archaeological Reports, 2001); Gerhard Bosinski, Die Ausgrabungen in Gönnersdorf 19681976 und die Siedlungsbefunde der Grabung 1968 (Wiesbaden: Steiner, 1979), and Tierdarstellungen von Gönnersdorf: Nachträge zu Mammut und Pferde sowie die übrigen Tierdarstellungen (Mainz: Verlag des Römisch-Germanischen Zentralmuseums, 2008); Gerhard Bosinski and Gisela Fischer, Die Menschendarstellungen von Gönnersdorf (Wiesbaden: Steiner, 1974), and Mammut und Pferdedarstellungen von Gönnersdorf (Wiesbaden: Steiner, 1980). I discuss these and similar materials (such as the engravings in Upper Paleolithic caves of Franco-Cantabria) in Replications: Archaeology, Art History, Psychoanalysis (University Park, PA: Pennsylvania State University Press, 1996), and 'Prehistoric Palimpsests', in Visuality and Virtuality: Images and Pictures from Ancient Egypt to New Media (forthcoming).

12 See especially Hallam L. Movius and Sheldon Judson, The Rock Shelter of La Colombière (Cambridge, MA: Peabody Museum of Archaeology and Ethnology, Harvard University, 1956). (This publication helped stimulate the controversial researches of Alexander Marshack in The Roots of Civilization: The Cognitive Beginnings of Man's First Art, Symbol, and Notation [New York: McGraw-Hill, 1972; second edition, Mount Kisco, NY: Moyer Bell, 1991], for which see Davis, A General Theory of Visual Culture, 128-49.) Many plaquettes were found broken in the rock shelters where they were excavated. But surprisingly few of the fragments can be put together. Therefore the breakage mostly occurred elsewhere; only some of the broken pieces were transported into the shelters, whether for use as 'broken' engraved plaquettes or for another use (for example, as flooring). Wherever it occurred, breakage does not seem to have been due to trampling or frost action; there is too much of it to suggest that it was accidental. Therefore we can incorporate deliberate breakage into the primary situation of use, and, as I prefer, into the use-meaning itself; to use the engravings was often to break them, and maybe to put them back together or to imagine new or other completions and to find them materially and visually in the accumulated corpus.

13 See Pâles and Tassin de Saint Péreuse, Gravures de la Marche, fig. 22, identifying 
a pictorialization of a particular feline (not identifiable zoologically as any real hunting cat) replicated on four different plaquettes (pls. 19, 20, 32, and 36).

14 See Bosinski, Tierdarstellungen von Gönnersdorf, Abb. 2, 6.

15 See Bosinski, Tierdarstellungen von Gönnersdorf, Abb. 11. In presenting this observation to audiences in North America and Europe, I find that some beholders can see the emergent head right away and without difficulty, and had noticed it even before I mentioned it verbally. Others, however, find it difficult to see even when its parts are described in relation to other visible figures. 